\title{
VIBRATION ANALYSIS AND DIAGNOSTICS FOR OIL PRODUCTION UNITS BY PUMPING ROD
}

\author{
Avram Lazar', Marius Stan², Maria Papadopoulou-Bakali ${ }^{3}$ \\ ${ }^{1}$ Department Driilig Production, Oil Gas University in Ploiesti, Romania, ${ }^{2}$ Department, Mechanical Engineering, Oil Gas \\ University in Ploiesti, Romania, ${ }^{3}$ Maria Rapadopoulos, Kavala Institute of Technology, Greece, \\ lavram@upg-ploiesti.ro,mstan@upg-ploiesti.ro,papa.maria2@hotmail.com
}

\begin{abstract}
Operating status of equipment can be appreciated in mechanical vibration analysis products and their parameters change over time. The paper presents methods of making a real analysis of oil extraction plant and how to interpret the results.
\end{abstract}

Index Terms:analysis, structure, vibration

\section{GENERAL}

Concrete manifestation mode of the vibration determined by, its various characteristics (displacements, velocities, acceleration, evolution in time, frequency or amplitude spectrum, etc...) Is individualized depending on the structure and the constructive and functional features of the system established by designed and built by execution, and in relation to operational status, performance, and any defects.

Vibration diagnostics of the state of operation of facilities, equipment and machinery is based on the fact that the energy transfer process, oriented towards a given function, different components, located on the route of the transfer, can be excited mechanically, and thus the vibration.

How long the process takes place constantly and consistently manifests excited vibration components. Indeed, any change in the operating state of dynamic change system default conditions, the conditions of propagation of elastic waves and vibration characteristics of the course and appreciated way or another.

Transmitted to the outside, route mobile organ-organ-supports fixed, the change forces, specific operation, is recorded, usually on the outside, the vibration and measure the relative motion of either component or as absolute motion.

In principle, the vibration can be considered as the result of the report, force to impedance mechanical result vibration.That the vibration change as a result of changes in operating status, force variation is essential. At the same time, although it is considered that the impedance is more stable factor is observed that the impedance depends on the particular structure and system elements. Thus, a tree moving in a camp, the impedance is dominated by the film of lubricant properties, while the same motion as measured on the outer surface of the bearings depends on the dynamic properties of the film, but at a much higher and the rigid body construction of the camp. Vibration measured at the outer surface of the bearings, will therefore be several times [2-4] less than estimated vibration directly, by moving the shaft in the bearing zone. How the operation of a machine does not work a single force as a source of vibration and elastic waves as the transfer can be done in several ways, it is obvious that the vibration response to various requests can become very complicated. Interpretation requires not only experience, data and information on features satisfactory vibration of the machine, but also the application of selection criteria and assessing the coincidence calculation - experiment.

\section{VIBRATION MONITORING AND DIAGNOSIS}

Surveillance equipment in operation and exploitation machines specific process operating parameters, vibration, temperature, etc... Is recognized as an important way to increase the reliability, operating efficiency, reduce production and operating costs. For use or installation of monitoring systems is to check normality operation, to detect deviations potential "problems" and to provide information, decision support and interventions to disconnect or shutdown and for diagnosis.

A series of possible failures in the operation of oil extraction plants, covered monitoring is indicated in Table 1, in connection with their development or evolution of the entire system for the damage and disruption. It should be noted that methodological separation gradual failure sudden failure time depends not only objective but also the processing of data for monitoring the conditions of the system, etc. surveillance by man. Thus, a sudden failure can occur in seconds, but in hours, a gradual failure in minutes but in months. 
Table-1 Possible failures in the operation of machinery

\begin{tabular}{|l|l|}
\hline \multicolumn{1}{|c|}{ Gradual failure } & \multicolumn{1}{|c|}{ Sudden failure } \\
\hline Attrition - balancing change & Friction parts; \\
Attrition - Change the alignment of bearings & Axial bearing failure; \\
Attrition - play in bearings and bearings increase, & The lack of lubricant in the bearings; \\
operating vibration; & Disruption of cooling; \\
The gears, operating the vibration; & Failure of turbine or compressor blades; \\
Fixed leaks in seals; & Dynamic instability; \\
Seals leaks in mobile contact (pistons, etc..) & The presence of foreign objects; \\
Slow-moving cracks in rotating elements. & Sealing fluid leaks fixed. \\
\hline
\end{tabular}

\section{SOURCES OF VIBRATION AND NOISE GEARS}

Usually, gears are the most important sources of noise and vibration from mechanical machinery structure, with a significant weight in determining the overall noise and vibration. The strong growth in power and speed of modern machines, while reducing gauge, may cause a worsening of behavior vibroacustice gear transmissions, especially when in the design, construction and installation were not pursued in this optimization criteria of view.
To adopt appropriate measures against vibration and noise transmission through the gears must be made acoustic behavior analysis simultaneously with the vibration behavior analysis.

Priority Way vibration and noise control measures a constiutie active protection because if a reduced dynamic response increases the durability of components (elements to reduce wear and fatigue relative moving equipment or destruction of dynamic overload) and at the same time increase the effectiveness of passive protection, Tab.2.

Table2. Constructive influence on the noise parameters

\begin{tabular}{|l|l|l|}
\hline Parameter analyzed & Range of values considered & $\begin{array}{l}\text { Increasing influence on the noise } \\
\text { parameter value }\end{array}$ \\
\hline Tooth inclination & $0 \mathrm{o} \div 40 \mathrm{o}$ & $\downarrow 20 \mathrm{~dB}$ \\
\hline Material stiffness & $\div$ & $\uparrow 12 \mathrm{~dB}$ \\
\hline Tooth flank & $(0 \div 0,02) \mathrm{mm}$ & $\downarrow 6 \mathrm{~dB}$ \\
\hline Coverage & $1 \div 2,2$ & $\downarrow 3 \mathrm{~dB}$ \\
\hline Modulul & $(1 \div 12) \mathrm{mm}$ & $\uparrow 2 \mathrm{~dB}$ \\
\hline Number of teeth & $\mathrm{z} \div 2 \mathrm{z}$ & $\uparrow 3 \mathrm{~dB}$ \\
\hline Specific displacement profile & $0,092 \div 0,883$ & $\downarrow \downarrow \mathrm{dB}$ \\
\hline Tooth width & - & $\downarrow$ little effect \\
\hline Curved tooth & - & $\downarrow$ The large errors of direction of \\
the tooth
\end{tabular}

\section{DIAGNOSIS BEARINGS}

Bearing, considered separately, the level of vibration and noise is one of the global indicators of quality, reflecting the effectiveness of specific technologies and installation finish. On the other hand, the level of noise vibraţiişi bearings mounted in a machine or equipment to an extent significant influence, along with other performance, product competitiveness in many applications (appliances, office and computer equipment with high precision cinematic). In operation, noise and vibration of bearings mounted correlated with their state of failure, indicating the presence of possibly increasing the fault and allowing such interventionsto avoid costly accidents Fig. 1 .. 


\section{Vibration}

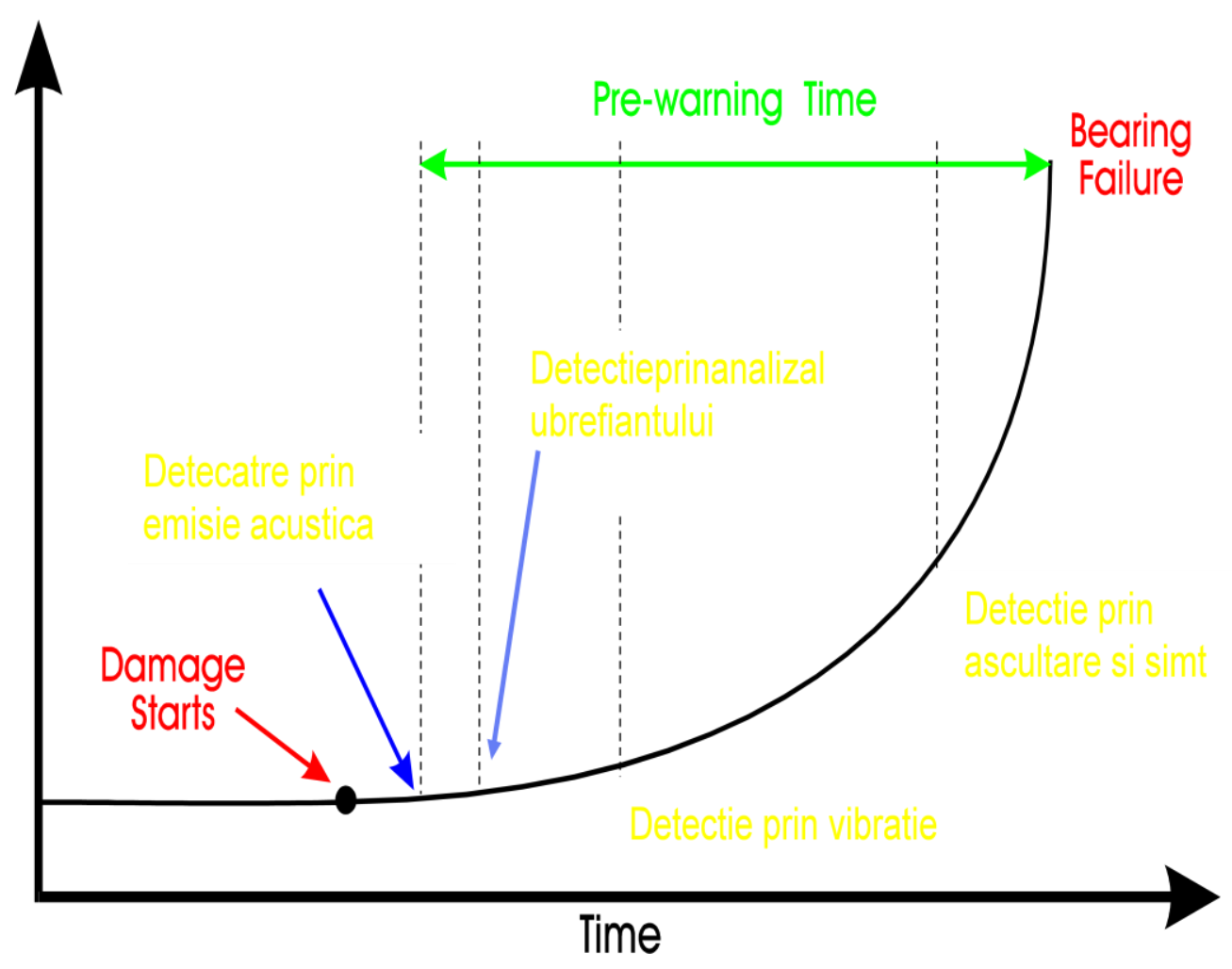

Fig.1. Typical failure rate of bearings

Bearing vibration and noise caused by acts or by direct effects, sound or vibration radiation, or by indirect effects. Direct effects is found especially in terms of technological research and testing on appropriate stands. Mounted bearings are used in machinery or equipment in a whole, in these conditions, there is both direct effects and, to a greater extent, indirect effects the other elements (trees, housing, etc.) or kinematic couplings, excited by vibration bearings, source path receiver. In bearings, vibration and noise generation has the following causes:

- Change position in the loaded rolling bodies, namely the unposted bearing about the size and number of rolling bodies, elastic deformations of contact work and play, while changing the position of the contacts, load, periodically changes the contact stiffness and the system, favoring the occurrence of type vibration parameters;

- Uneven movement of rolling bodies, due to different applications depending on position, this movement also lead to uneven friction and collisions of bodies rolling with rings or cage;

- Establish contacts with rolling surfaces with deviations dimensional form andPosition: differences in diameter, front or radial beat taxiways, eccentric, oval, poligonalitate, corrugations, roughness;
Moving bodies rolling over the buttons placed impurities or defects or damage over localized (pinching type peeling or pitting, abrasive wear, fingerprints, etc.).

\section{MEASURING VIBRATIONS IN THE MAIN CAMPS OF PUMPING UNIT UP 5T}

The device used for measuring vibration from pumping unit 5T UP $1500-1000 \mathrm{C}$ is called VIBROTEST 60, produced by German fimaBrüel\&Kjær, Schenck.

VIBROTEST 60 is specially designed for vibration measurements, spectra, and process variable data values over time and is designed to last the technological solutions.

Rules for correct measurement:

- Always on camps;

- Measure the three directions $(\mathrm{H}, \mathrm{V}, \mathrm{A})$;

- The sensor firmly attached to the camp;

- Using the same numbering system for all equipment measured bearings;

- Using the appropriate set of measurement type. 


\begin{tabular}{|l|c|c|c|}
\hline & $\begin{array}{c}\text { Speedinput } \\
\text { rpm }\end{array}$ & $\begin{array}{c}\text { Vibrations - V } \\
\text { rms m/s }\end{array}$ & $\begin{array}{c}\text { Vibrations - H } \\
\text { rms m/s }\end{array}$ \\
\hline L1 & 490 & 0,121 & 0,074 \\
\hline L2 & 490 & 0,185 & 0,105 \\
\hline L3 & 490 & 0,145 & - \\
\hline L4 & 490 & 0,162 & - \\
\hline L5 & 490 & 0,117 & 0,125 \\
\hline L6 & 490 & 0,128 & 0,124 \\
\hline LC & 490 & 0,107 & 0,135 \\
\hline M & 490 & 1,109 & 1,080 \\
\hline
\end{tabular}

Table 3.1Vibration the main campus UP 5T.

\begin{tabular}{|l|l|l|}
\hline & $\begin{array}{l}\text { Speed input } \\
\text { rpm }\end{array}$ & $\begin{array}{l}\text { Vibrations - V } \\
\text { Rms in m/s }\end{array}$ \\
\hline L1 & 110 & 0,035 \\
\hline L2 & 127 & 0,031 \\
\hline L3 & 204 & 0,033 \\
\hline L4 & 137 & 0,110 \\
\hline L5 & 148 & 0,106 \\
\hline L6 & 182 & 0,080 \\
\hline
\end{tabular}

Table3.2 Vibration when braking of main bearings UP 5T

\section{VIBRATION ANALYSIS OF CENTRIFUGAL}

\section{PUMPS}

The pump is a machine which converts mechanical energy received from a driving source (for example, an electric motor) hydraulic energy. Transport of the liquid by the pump can only be achieved in a hydraulic system. Such a system typically consists of: pipes, fittings, meters and of course the pump, as the main element.

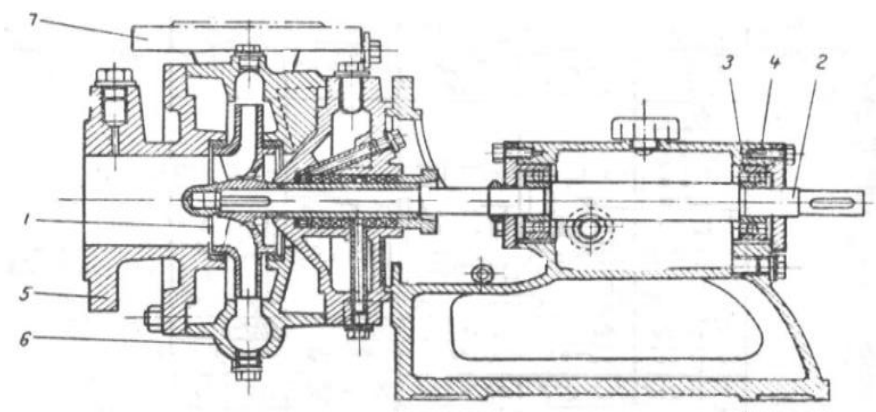

Fig. 4 Construction of centrifugal pumps

Fluid enters the pump through the conduit aspiraţie1. The rotor 2 is fixed to the arborele3. By turning the rotor is spun on its outskirts water where the water is collected in room 6

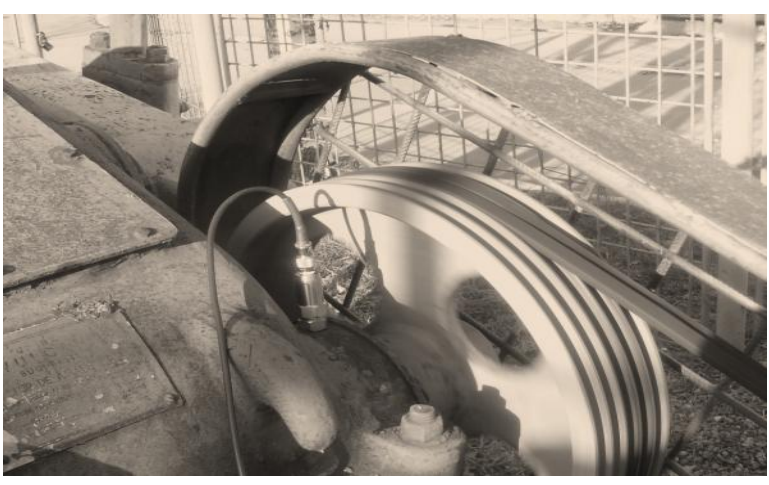

Fig2. Position measuring sensors main UP 5T

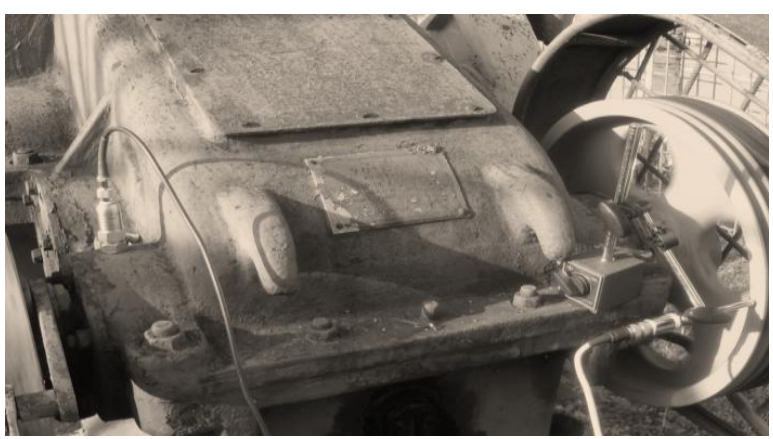

Fig 3 Position measuring sensors braking of UP 5T

and headed to discharge spiral 7 in speaker 5 . The water exits through the high-pressure pipe 4 in Figure 4.Causes wear

Functional parameters of a pump do not keep constant values throughout her life. This is explained by the fact that the parts which comprise pump wear over time. If nature centrifugal pumps wear parts is of two types:

- Mechanical;

- Cleaning.

Whatever the nature of wear, it has the effect of changing geometric shapes of the parts, which are ultimately reflected in the modification of the hydraulic pump functional parameters (flow, pressure, pump head)

Assemblies that wear out frequentlyCentrifugal pumps, parts most exposed to wear, both abrasive and corrosive are:

- Rotor;

- Bearings and measuring point 1,2 ;

- The shaft protection sleeve;

- Mechanical seal;

- Soft linings;

- Camps.

During unit shutdown to perform maintenance stage wear is found and pass the parts replaced. For vibration analysis VIBROTEST we use the data collector device 60 being at the same time balancing tool, Figure 6. 


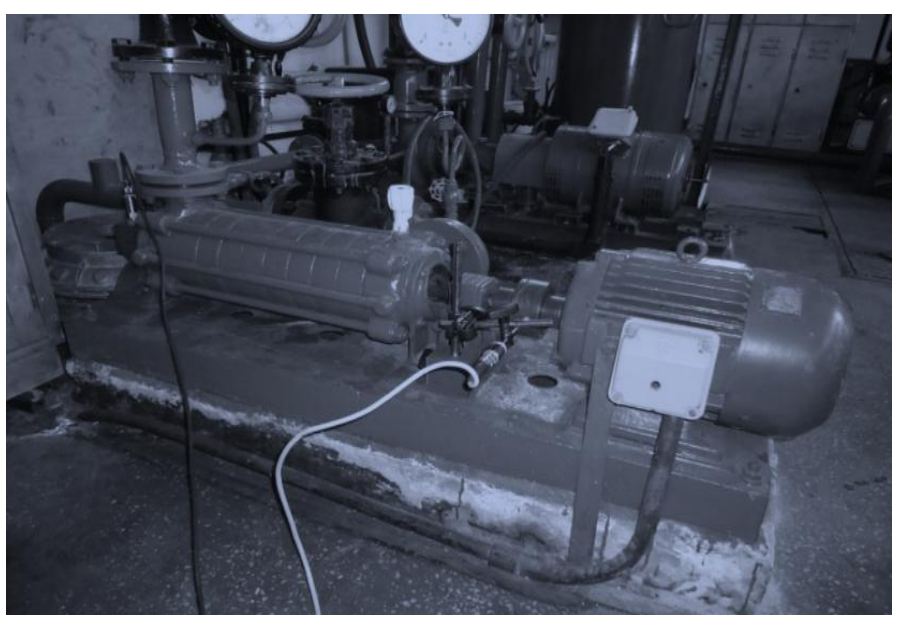

Fig6. RMS vibration measuring their various multistage centrifugal pumps

Rules Concerning the correct measurement:

Always on bearings;

Is Measured in three directions $(\mathrm{H}, \mathrm{V}, \mathrm{A})$;

The sensor firmly attached to the field;

Using the same numbering system on all machines measured bearing;

Using the setting for the type of measurement. When it is found that the vibration level reached in the "more acceptable" maintenance team is warned. When the vibrations exceed the maximum permissible value is necessary to stop the machine, its removal to determine the causes of vibration and then repair the fault.

Preventive maintenance involves periodic measurements of vibration and noise from the bearings and comparing the measured values with the prescribed equipment manufacturer or standards.

Predictive maintenance is a qualitative leap higher in modern maintenance systems, regardless of the specific industry or production, as it provides all information necessary to: earlier detection of faults occur, their location, fault diagnosis, lifetime calculation in safe equipment.

Table4. Experimental results from the analysis of vibrations multistage centrifugal pumps

\begin{tabular}{|l|l|l|l|l|l|l|}
\hline $\begin{array}{l}\text { Discharge } \\
\begin{array}{l}\text { Pressure } \\
{[\mathrm{kPa}]}\end{array}\end{array}$ & \multicolumn{3}{|l|}{ BEARING 1 (speead 2960 rot/min) } & \multicolumn{3}{|l|}{ BEARING 2 (turatie 2960 rot/min) } \\
\cline { 2 - 7 } & $\begin{array}{l}\text { orizontal } \\
{[\mathrm{mm} / \mathrm{s}]}\end{array}$ & $\begin{array}{l}\text { vertical } \\
{[\mathrm{mm} / \mathrm{s}]}\end{array}$ & $\begin{array}{l}\text { axial } \\
{[\mathrm{mm} / \mathrm{s}]}\end{array}$ & $\begin{array}{l}\text { orizontal } \\
{[\mathrm{mm} / \mathrm{s}]}\end{array}$ & $\begin{array}{l}\text { vertical } \\
{[\mathrm{mm} / \mathrm{s}]}\end{array}$ & $\begin{array}{l}\text { axial } \\
{[\mathrm{mm} / \mathrm{s}]}\end{array}$ \\
\hline 1 & 0.180 & 0.284 & 0.148 & 0.143 & 0.211 & 0.098 \\
\hline 0.8 & 0.249 & 0.410 & 0.257 & 0.130 & 0.139 & 0.119 \\
\hline 0.6 & 0.292 & 0.315 & 0.236 & 0.133 & 0.134 & 0.131 \\
\hline 0.3 & 0.267 & 0.315 & 0.269 & 0.121 & 0.123 & 0.139 \\
\hline
\end{tabular}

\subsection{Interpretation of Results}

Vibration measurement will indicate if the vibration rms is normal, still admissible or inadmissible, but we will provide information on what is defective and where it is located. Their values, compared with the permissible levels recommended by the manufacturer or dynamic machine ISO 2372 and ISO 10816, figure 4, and indicate that the machine works safely. 


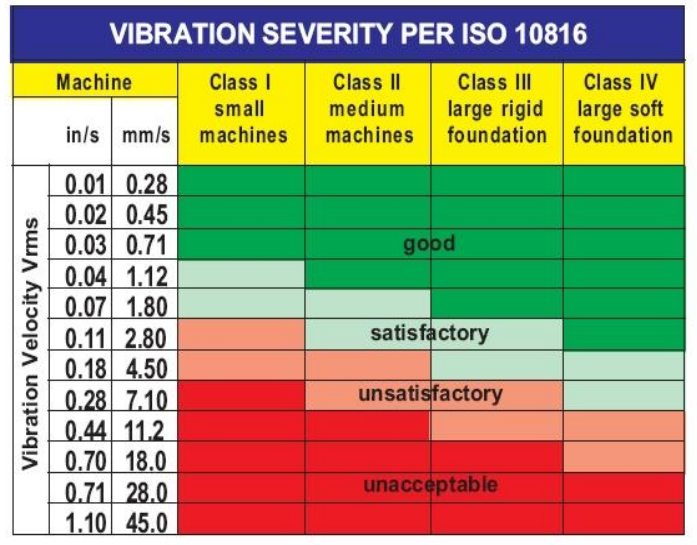

Fig.7 Machine works safely

The result is the assurance of design integrity prior to equipment installation and use. MechRel then extends these cost savings to actual equipment usage through early identification of an optimum maintenance philosophy;logistics support requirements and real time calculations of remaining life.

MechRel is used to perform different types of reliability analyses of mechanical designs and determine logistics support requirements. Using MechRel saves considerable expense during the early development phase by determining the reliability, availability and maintainability characteristics long before the expensive "test analyze and fix" phase of design development.

MechRel is an enhancement to any platform analysis. It provides a more accurate determination of failure event probabilities, probabilities of occurrence for individual failure modes, and supports the transition from hard time maintenance to conditioning monitoring and condition based maintenance. For example, CDNSWC engineers have demonstrated the use of MechRel remaining life equations for an automated diagnostic and prognostic system.

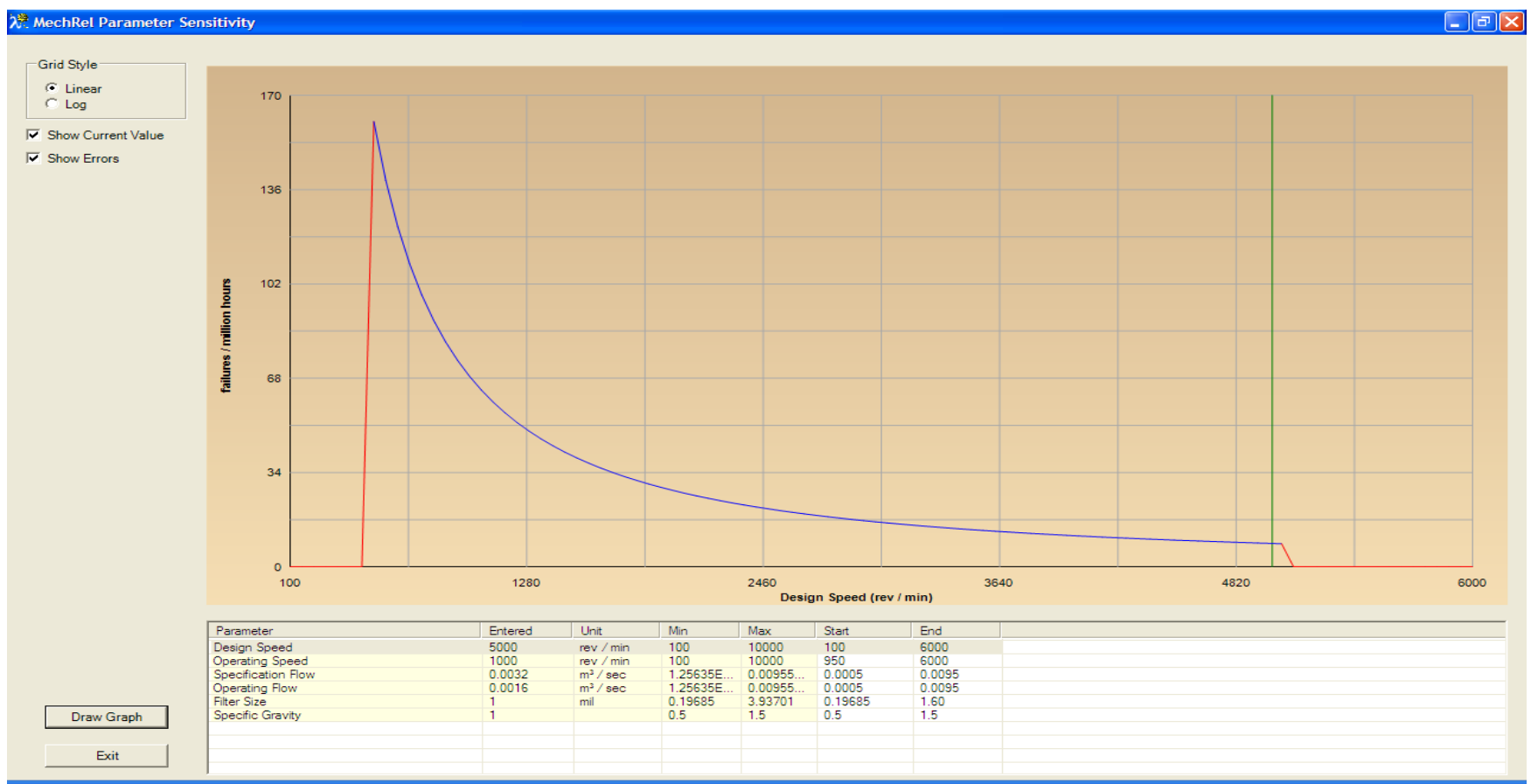

Fig8 Failures / million hours function according design speed

\section{CONCLUSIONS}

Centrifugal pumps are machines that convert mechanical energy into hydraulic energy by the active part (rotor), with different structural forms: single-stage, multistage horizontal or vertical multistage multistage oblique.

The main causes of wear centrifugal pumps may be of mechanical or chemical nature and the parts most affected are: rotor, bearings, shaft protection sleeve, soft gaskets, and mechanical seals labirintii.

Vibrations caused by mechanical weakening trees are the most dangerous because they lead to additional demands by shock shaft and bearings. 
Diagnosis of mechanical loosening vibration analysis method has several advantages such as reducing maintenance costs, increased operational safety, reduce wear in bearings etc.

Vibration on bearings is one of the global indicators of quality, they manifested either by direct effects or by indirect effects. With measurements of vibration can cause defects such as dynamic imbalance, poor alignment, games and weakening, specific defects of bearings, etc.

In vibration analysis performed on three types of centrifugal pumps, namely: single-stage pump, horizontal multistage pumps with four rotors and ten horizontal multistage pump impellers, it follows that these machines do not require repair surgery because the vibrations are within the limits prescribed.

Diagnosis of mechanical loosening vibration analysis method has some advantages such as lower maintenance costs, increased operational safety strain relief camps etc.

In vibration analysis performed on three types of centrifugal pumps, namely: single-stage pump, horizontal multistage pump with four rotors and ten horizontal multistage pump impellers, that these machines do not require repair job because the vibrations are within the prescribed limits.An analysis of a design for reliability and maintainability identifies critical failure modes and causes of unreliability, and provides an effective tool for designing the most cost effective logistics support plan for the product.

Applying MechRel to a new product design provides a sound basis for determining spare parts requirements, needed redesign efforts, and reallocation of logistics resources to assure that $\mathrm{R} \& \mathrm{M}$ requirements for a particular system will be met.MechRel is an enhancement to any platform analysis. It provides a more accurate determination of failure event probabilities, probabilities of occurrence for individual failure modes, and supports the transition from hard time maintenance to conditioning monitoring and condition based maintenance. For example, CDNSWC engineers have demonstrated the use of MechRel remaining life equations for an automated diagnostic and prognostic system.

\section{REFERENCES}

[1]Giuseppe Aiello, s.a. Real time assessment of hand-arm vibration system based on capacitive MEMS accelerometers, Computers and Electronics in Agriculture, Volume 85, July 2012

[2] Stan Marius, Fiabilitateasistemelorsiaplicatii, EdituraUniversităţii Petrol - Gaze din Ploieşti, ISBN 978-973719-249-3, 2010

[3]Stan Marius, Analysis the significance of reliable experimentally determined distribution laws, 3 rd Symposium with international participation Durability and Reliability of Mechanical Systems, Targu-Jiu,ISBN 978-973-144-350-8, Mai, 20-21 2010
[4]Mechanical Reliability Prediction Software Package, Carderock Division, NAVSEA 